\title{
Signal local Reconstruction Algorithm based on Compressed Sensing and Unsupervised Learning
}

\author{
Lingli TAN ${ }^{1, \text { a }}$ \\ ${ }^{1}$ School of Computer Science, Wuhan Donghu University, Wuhan, China, 430212
}

\begin{abstract}
Keywords: Signal Reconstruction; Sparsity; Compressed Sensing; Local Reconstruction; Unsupervised Learning
\end{abstract}

\begin{abstract}
With the rapid growth of data volume of the Internet and other platforms, the bandwidth needed for data transmission and reception is getting higher and higher, and the requirements for processing speed and sampling frequency of information acquisition are also improved. Based on the Shannon sampling theory, it is found that only when the sampling frequency of the signal is higher than or equal to twice the signal bandwidth, can higher-quality analog signal recovery effect be achieved. In order to efficiently deal with the problem of the fast reconstruction of the unknown sparsity of the compressed signal, a new method with wider adaptability and higher efficiency is proposed. Firstly, use isometric rules to obtain upper and lower bounds of the compressed output signal, and take the closest integer value as estimation value of sparse signal; secondly, by reducing the number of iterative projection support of observation vector to realize complexity reduction of calculation of signal reconstruction, and design evaluation probability system for signal reconstruction to achieve implementation of the validation of the proposed index scheme; finally, based on the experimental verification, the proposed method can obtain and achieve fast reconstruction of sparsity of unknown signal and can obtain higher success rate than backtracking scheme.
\end{abstract}

\section{Introduction}

Danoho, Candes and other experts found and interpreted the process of compressed sensing of information based on research [1-3]. The main idea of this paper is to reduce the computational complexity of the algorithm starting from the simplified reconstruction projection process by using the compressed sensing process. In order to solve the problems mentioned above, this paper designs a new method of signal reconstruction (OASP) which is more adaptive and efficient. In this method, the sparse prediction algorithm is used to achieve reconstruction of the information of unknown sparsity. At the same time, it can effectively avoid the redundant computation of the iterative support set projection of the observation vector in the reconstruction process to reduce the computational complexity of the reconfiguration strategy. Then, evaluation index design of the performance of reconstruction of information on the basis of in-depth study of the existing methods of measuring is performed to get access to a more scientific and comprehensive assessment. The experimental analysis shows that the sampling frequency standard of the process is much lower than the Nyquist standard, and the original signal reconstruction results obtained by this method have higher reliability.

\section{Strategy Description}

As previously mentioned, because the present reconstruction strategy only applies to reconstruction of signal with known sparsity, but not applicable to signal with unknown sparsity, this paper makes improvement on reconstruction strategy to combine sparsity fast estimation strategy for implementation of efficiency promotion of calculation process of sparsity and realization of unbiased sparsity estimation. In addition, in the proposed reconstruction strategy, projection process of observation vector iterative candidate set of backtracking strategy is improved to obtain sufficient convergence and avoid the support set projection calculation, so as to realize the simplification of reconstruction process. 


\section{(1) Sparsity estimation}

Here, the adopted estimation strategy is firstly described. The fact that the signal with unknown sparsity to be reconstructed meets isometric constraint conditions [6] is the premise for reconstruction. According to this property, in case the vector $x$ satisfies the condition $\|x\|_{0} \leq K$, if there is a matrix $A$ that satisfies the condition of the formula:

$$
\left(1-\delta_{K}\right)\|x\|_{2}^{2} \leq\|A x\|_{2}^{2} \leq\left(1+\delta_{K}\right)\|x\|_{2}^{2}
$$

Then the matrix A satisfies the constraint conditions in case of parameters $(K, \delta)$. In formula (1), $\|x\|_{2}$ is the $l_{2}$-norm result of $x$, in which $\delta_{K}$ is the minimum condition available that satisfies the formula (1), that is, $0<\delta_{K}<1$. Theorem 1 can be obtained using the above properties.

Theorem 1: set the sparsity value of the signal to be reconstructed as $k$, for the parameters $(K, \delta)$, the matrix A meets the constrained isometric characteristics, if the equation $k=K$ is established, then the following formula can be obtained:

$$
\frac{1-\delta_{k}}{\sqrt{1+\delta_{k}}}\|y\|_{2} \leq\left\|A_{10}^{T} y\right\|_{2} \leq \frac{1+\delta_{k}}{\sqrt{1-\delta_{k}}}\|y\|_{2}
$$

In the formula (2), $\left|\left(A_{i}, y\right)\right|$ is the absolute value of the vector $y$ and the matrix $A$ of elements in $i$ th column, $\Gamma_{0}$ is the set of maximum values of the previous $k$ sets of indexes, and $A_{r_{0}}$ is information sub matrix constructed in $\Gamma_{0}$ th column in matrix $A$.

Proof:

Lemma 1: set the sparsity of the signal to be reconstructed as $k$, for the parameters $(K, \delta)$, the matrix Ameets the constrained isometric characteristics, if $k \geq K$ is established, then the following formula can be obtained:

$$
\frac{1-\delta_{k}}{\sqrt{1+\delta_{k}}}\|y\|_{2} \leq\left\|\boldsymbol{A}_{r_{0}}^{T} y\right\|_{2}
$$

Lemma 2: if matrix A meets the constrained isometric characteristics as for parameters $(K, \delta)$ and meets $\left\|A_{i_{0}}^{T} y\right\|_{2}>\frac{1+\delta_{k}}{\sqrt{1-\delta_{k}}}\|y\|_{2}$, then it can be concluded that $k>K$.

According to the inverse-negative proposition of lemma 2, the following inference can be obtained:

Inference 1: if the matrix A satisfies RIP characteristics of parameter $\left(K, \delta_{K}\right)$ and satisfies the sparsity estimation characteristic of $\left\|A_{r_{0}}^{T} y\right\|_{2} \leq \frac{1+\delta_{k}}{\sqrt{1-\delta_{k}}}\|y\|_{2}$, then it can be concluded that $k \leq K$.

Theorem 1 can be approved by using inference 1 and lemma 1 .

$\frac{1-\delta_{k}}{\sqrt{1+\delta_{k}}}\|y\|_{2}$ and $\frac{1+\delta_{k}}{\sqrt{1-\delta_{k}}}\|y\|_{2}$ used in theorem 1 are constant terms greater than zero, set the two items as $C_{\min }$ and $C_{\max }$ and calculate the square of both sides of formula (2), then it can be obtained:

$$
C_{\min }^{2} \leq \sum_{i \in}\left(\mid\left\langle A_{i}, y\right\rangle\right)^{2} \leq C_{\max }^{2}
$$

Set $u_{i}=\left\langle\left\langle A_{i}, y\right\rangle\right|(1 \leq i \leq N)$, arrange $u_{i}$ in descending order to obtain the new data $\operatorname{set}^{\prime}=\left(u_{1}^{\prime}, \cdots, u_{i}^{\prime}, \cdots, u_{N}^{\prime}\right)$. Set $f(k)=\sum_{i=1}^{k} u_{i}^{2}$, then formula (3) is equivalent to:

$$
C_{\min }^{2} \leq f(k) \leq C_{\max }^{2}
$$

In formula (4), the minimum $k$ value can be used as the lower limit of sparsity $K$, and it can be expressed as $K_{\min }$, the maximum ${ }^{k}$ value that makes formula (4) hold is:

$$
k=\left\lfloor 0.5\left(K_{\min }+K_{\max }\right)+0.5\right\rfloor
$$

The symbol \lfloor\rfloor in formula (4) means rounding operation of that under the symbol.

Compared with traditional methods [11-12], it is only needed to calculate the square sum of the internal elements of $U^{\prime}$, express respectively the first and the last element satisfying the formula (4) as $K_{\min }$ and $K_{\max }$ to avoid the redundant calculation. in related literatures [11-12], stop signal reconstruction if the sparsity value is in interval of the upper and lower limits, for it can lead to excessive error of sparsity estimation value, for example, when testing at the position of lower limit of sparsity but the real value is in the upper limit. This paper adopts $k=\left\lfloor 0.5\left(K_{\min }+K_{\max }\right)+0.5\right\rfloor$, which can effectively avoid above situation, and can obtain more accurate estimated value. 


\section{(2) Algorithm steps}

The implementation process of the proposed reconstruction strategy is shown in Figure 1. Under the premise of ensuring backtracking reconstruction strategy satisfies the convergence conditions, the reduction of projection calculation times of reconstruction strategy can efficiently reduce complexity of the strategy. Firstly, the $K$ value is estimated by the estimation method, and then the iterative process of signal reconstruction is performed. For $n$ times of signal reconstruction iterative process, firstly compute untreated margin $r_{n-1}$ and matrix $A$ according to the column correlation, merge $K$ groups of indexes of strongest column correlation coefficient and $S_{n}-1$ obtained in the last iteration process, and then get $C_{n}$, then perform projection operation of $y$ in $C_{n}$ and set $K$ groups of indexes of strongest column correlation coefficient as $S_{n}$, and set $S_{n}$ projection as non zero subentry $Z s_{n}$ of $Z_{n}$ of signal reconstruction iterative process; in order to achieve effective iterative margin determination, $r_{n}=y-A_{s_{n}} \cdot Z_{S_{n}}$, if the condition $\left|r_{n}\right|>\left|r_{n}-1\right|$ is satisfied, then stop signal iteration, if the above conditions are not satisfied, proceed to the next iteration, among which $A s_{n}$ is the sub matrix of the index column $S_{n}$ in the matrix $A$. The calculation process is shown in Figure 1.

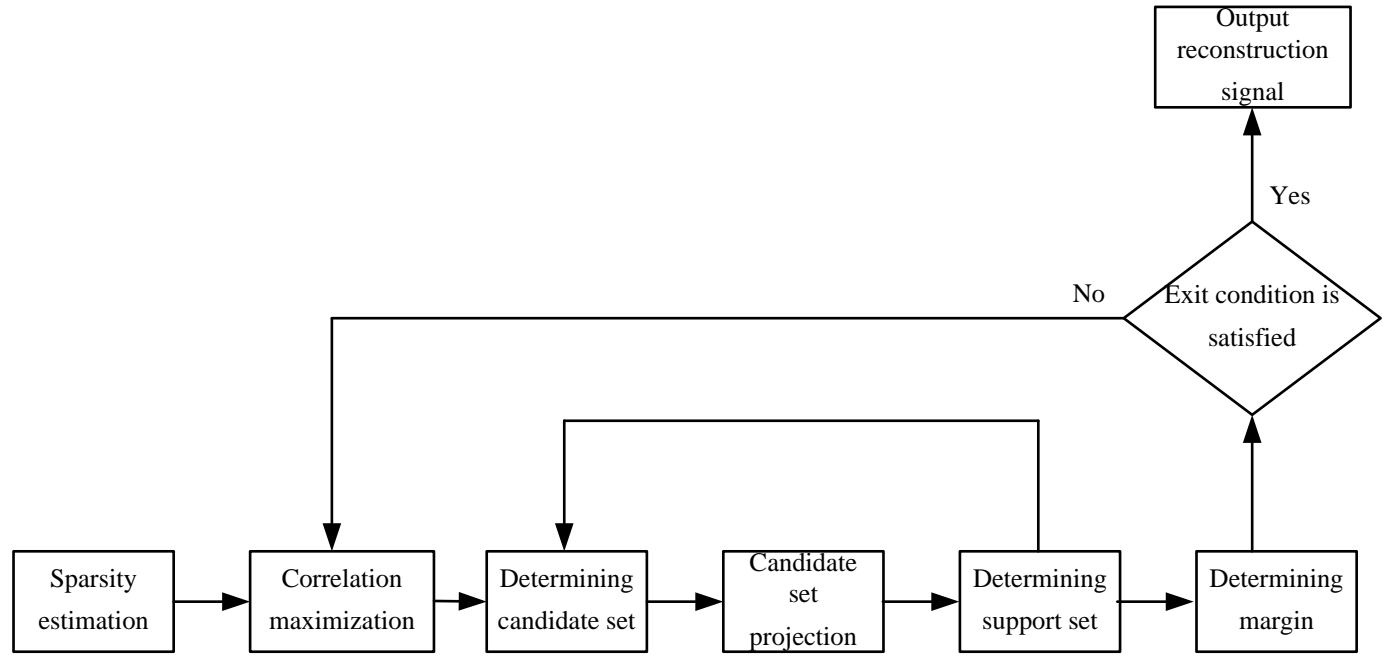

Fig.1. Method implementation steps

\section{(3) Theoretical analysis}

Theorem 2: suppose that the signal $x \in R^{N}$, the true sparsity $K \leq M / 3$, the observation values of the signal $y=\Phi_{x} \in R^{M}$, if $\Phi$ satisfies RIP characteristics of the parameters $(K, \delta)$, and meets $\delta_{K}<0.206$, then the proposed algorithm can ensure ${ }^{y}$ can achieve reconstruction of ${ }^{x}$ sequence through limited iterative process, that is, reconstruction process convergence.

Proof: the proof of that the reconstruction process is convergent can be performed by proving each convergence of reconstruction process will cause the energy of signal sequence of energy to gradually reduce, that is: $\left\|x_{T-T^{l}}\right\|_{2} \leq C \cdot\left\|x_{T-T^{l}-1}\right\|_{2}, 0<C<1$.

1) Simplification process of index definition projection process is $\Delta T=\tilde{T}^{l}-T^{l}$, then $\mid \pi_{\Delta T}\left(x_{p}\right)\left\|_{2} \leq \pi_{T}\left(x_{p}\right)\right\|_{2} \leq\|\varepsilon\|_{2}$, that is:

$$
\mid x_{\Delta T}\left\|_{2} \leq\right\| \pi_{\Delta T}\left(x_{p}\right)\left\|_{2}+\right\| \varepsilon \|_{2}
$$

If $x_{\Delta T}=x_{T \cap \Delta T}$, then:

$$
\left\|x_{T-T^{l}}\right\|_{2} \leq \frac{1+\delta_{K}}{1-\delta_{K}}\left\|x_{T-T} l\right\|_{2}
$$

2) Because: $\eta_{-1}=\Phi_{T \cup T}{ }^{l-1} x_{r}^{l-1}$ and property of RIP is:

$$
\left\|x_{p, T}^{l-1}\right\|_{2} \leq \frac{\delta_{S^{l-1}+K}}{1-S^{l-1}}\left\|x_{T-T^{l}}\right\|_{2}
$$

For the simplification $T_{\Delta}$ of the projection process, it can be obtained according to definition of $T_{\Delta}$ that:

$$
\mid \Phi_{T_{\Delta}}^{T} r_{l-1}\left\|_{2} \geq\right\| \Phi_{T}^{T} r_{l-1}\left\|_{2} \geq\right\| \Phi_{T-T^{l-1}}^{T} r_{l-1} \|_{2}
$$

It can be seen that simplifying the intersection part of $T_{\Delta}$ and $T-T^{l-1}$, the formula is still established: 


$$
\left\|\Phi_{T_{\Delta}-T^{T}}^{T} r-1\right\|_{2} \geq\left\|\Phi_{T-T^{l-1}}^{T} \eta_{-1}\right\|_{2}=\left\|\Phi_{T-\tilde{T}^{l}}^{T} r_{l-1}\right\|_{2}
$$

Thus each convergence in the rebuilding process will cause the energy of signal sequence to gradually reduce, that is, the equation $\left\|x_{T-T^{T}}\right\|_{2} \leq C \cdot\left\|x_{T-T^{l}-1}\right\|_{2}, 0<C<1$ is established.

\section{Reconstruction Evaluation Index}

In the sparse signal reconstruction operation, there will be the reconstruction errors of location reconstruction error and amplitude reconstruction error, the experimental data is shown in Figure 2, figure 2a is the original sparse signal without being processed, the signal length is 10 and the sparsity is 4; figure $2 \mathrm{~b}$ is sparse signal with amplitude reconstruction error, figure 2c is the sprsity signal with location reconstruction error. The probability of the non - zero amplitude of the signal is reconstructed, which is defined as I, and set the probability of sparse reconstruction as II . If $\mid$ Reconstructed signal-original signal $\mid \leqslant 10-8$ is satisfied, the reconstruction process is successful. The output of the signal reconstruction process is shown in figure $2 \mathrm{~b} \sim 2 \mathrm{c}$, and the corresponding values of the reconstruction probability are 0.75 and 0.9 respectively; In the traditional evaluation process, I is mainly taken as evaluation index to evaluate reconstruction performance of the proposed method. The reconstruction probability I can not reflect the difference between the two kinds of error reconstruction, and the simple using of reconstruction probability I can not judge the whole reconstruction probability of the sparse signal.

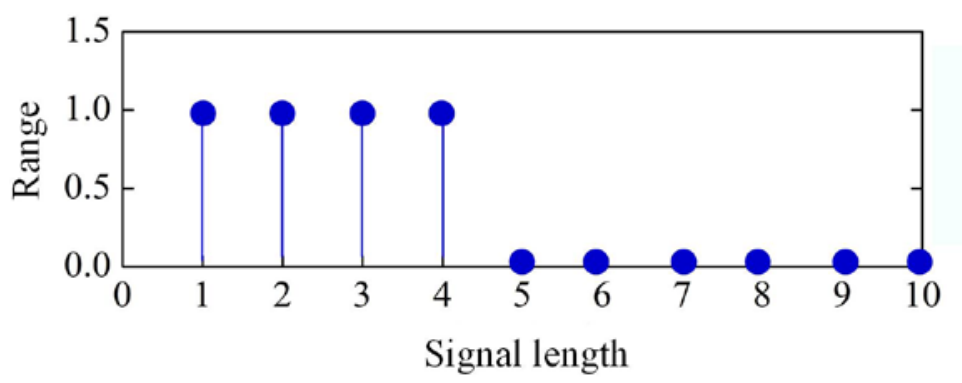

(a) Raw signal

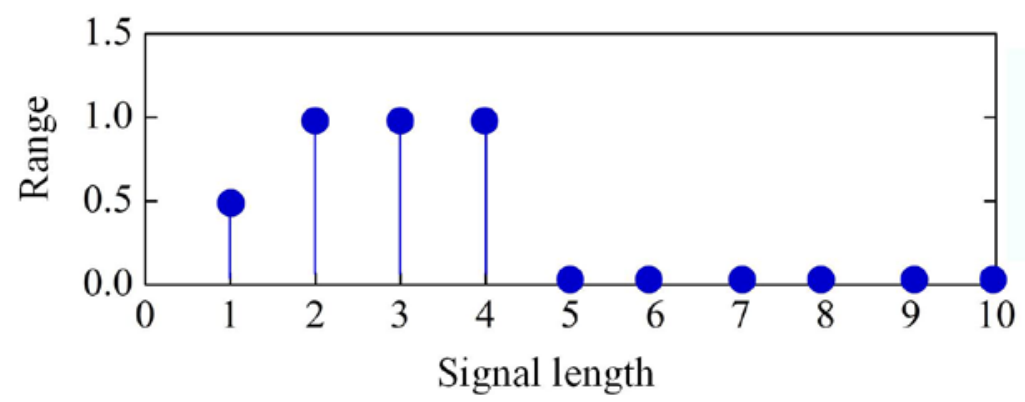

(b) Amplitude reconstruction error

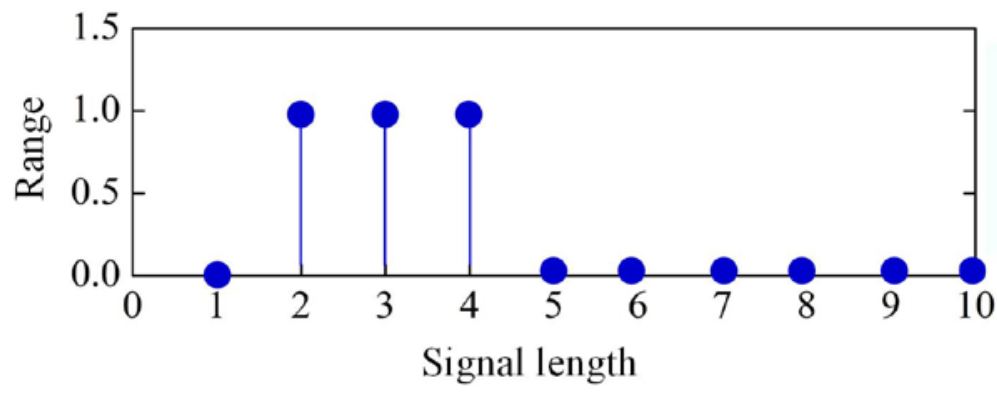

(c) Location reconstruction error

Fig.2. Signal reconstruction error

For the reconstruction process, the best effect is to achieve unbiased estimation of the entire signal sparse reconstruction, rather than simply perform the reconstruction process for the non-zero 
signal elements. In this paper, the performance of signal construction of the proposed method is analyzed by using the sparse reconstruction probability II as the evaluation index in the following simulation.

\section{Experimental Analysis}

\section{(1) Sparsity evaluation}

Determination is drawn based on accuracy estimation of sparsity by simulation, in order to simplify the description, here it is referred to as OASP. In the process of experiments, respectively set the length of the signal $\mathrm{N}$ as 256 and sampling frequency $\mathrm{M}$ as 128, the observation matrix used is $M \times N$ dimension random Gauss matrix, comparison of the experimental data used is mean of 1000 experiment stimulation statistical data. In Figure 3 , when $K=36$, by comparing the estimated $K$ values of each $\delta_{k}$ value, we can see that in the case that $\delta_{k}=0.15$, the estimated value of the strategy is smaller than the actual deviation value, and is more stable.

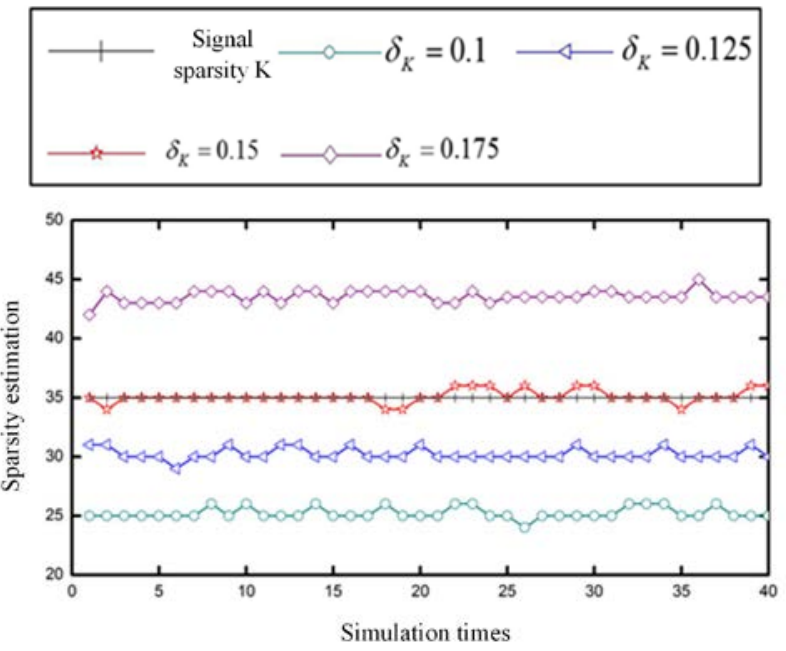

Fig.3. Experimental comparison of sparsity

Table 1 compares the simulation time of the two strategies. The experimental results are comparison simulation time of the two different strategies for 1000 times, which is the statistical data of 1000 Monte Carlo experiments. In the experiment, set $\mathrm{N}=512, \mathrm{M}=256$, compare the computation time for achieving signal reconstruction under the same constraints at the end of the experiment. Based on the data in the table, we can see that compared with the reconstruction strategies of OASP and $S P$, the computation time of the previous strategy is less than that of $S P$ reconstruction strategy, and with the increase of $K$ value, the decrease of computational time of OASP strategy is more obvious.

Table 1 Comparison of computation time

\begin{tabular}{|c|c|c|c|c|c|}
\hline \multirow{2}{*}{$K$} & \multicolumn{2}{|c|}{ Computation time/s } & \multirow{2}{*}{$K$} & \multicolumn{2}{c|}{ Computation time/s } \\
\cline { 2 - 3 } \cline { 5 - 6 } & SP & OASP & & SP & OASP \\
\hline 8 & 0.267 & 0.218 & 40 & 40 & 3.472 \\
\hline 16 & 0.771 & 0.606 & 48 & 48 & 5.029 \\
\hline 24 & 1.574 & 1.321 & 56 & 56 & 6.909 \\
\hline 32 & 2.651 & 2.129 & 64 & 64 & 9.687 \\
\hline
\end{tabular}

Figure 4 shows the number of iterations of the same set of termination conditions of the two strategies, set in the simulation process that $N=256, M=128$, the contrast data figure 4 shows is mean of 500 Monte Carlo experimental statistical data. Based on this figure, we can see that when the $K$ value is small, the number of iterations required by the contrast strategy in the same termination condition is not large, and its value is close to $\log (K)$. With the increasing of $\mathrm{K}$ value, the number of iteration steps of $O A S P$ strategy increases, but the $S P$ reconstruction strategy is contrary. With the increasing of $\mathrm{K}$ value, the saving time of OASP strategy is becoming more and more obvious. 


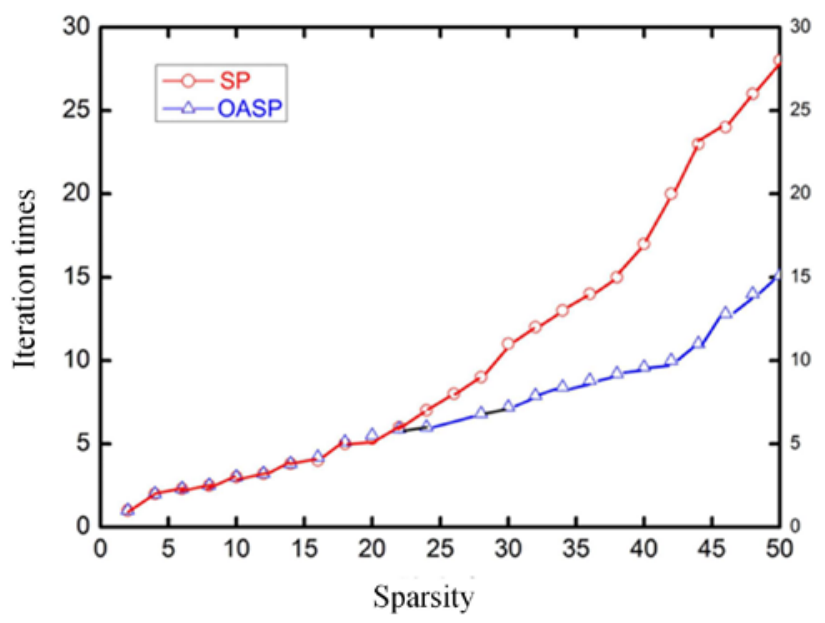

Fig.4. Comparison of iteration times

Figure 5 shows the comparison of the number of iterations under different reconstruction probability II . In simulation comparison, set $N=256, M=128$, as for $\mid$ Reconstructed signal-original signal $\mid \leqslant 10-12$, it indicates that the signal reconstruction process is successful, comparison data as shown is Monte Carlo mean of 1000 statistical data. At the same time, several strategies such as SAMP(Sparsity Adaptive Matching Pursuit) [15] are adopted for comparison. The results show that the performance of the three strategies of ROMP , OMP, SAMP decreases gradually under the situation that the sparsity is less than 45 . At the same time, the $S P$ strategy has better performance of signal reconstruction, but when $K$ is increased to 56 , the reconstruction performance of the strategy is beginning to decline; especially when the $K$ value of the OASP strategy reaches 60 , its performance begins to decline. This is because for each strategy, there is a sparse threshold, when the threshold is exceeded, the ability of the algorithm declines rapidly, resulting in the rapidly reduction of performance of the algorithm, which is as shown in figure 5. The greater the threshold value, the stronger the reliability of the algorithm, therefore, based on the above data, we can see that the proposed method has the most prominent advantages in the process of the reconstruction of the sparse signal, and its computational efficiency is the highest. Although the proposed method has a significant advantage, but with the increase of the $K$ value, the reconstruction probability II is greatly reduced, but the computation time of the SAMP strategy is much slower.

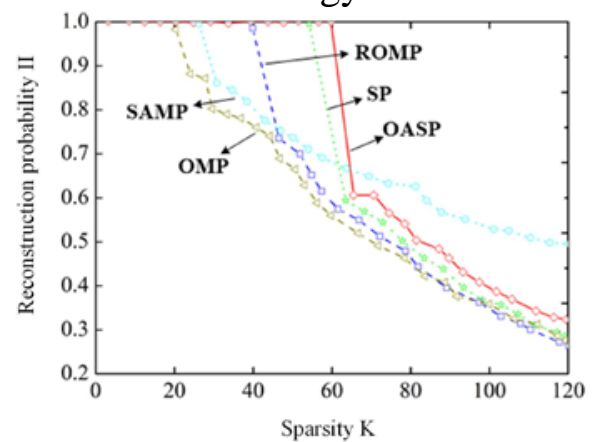

Fig.5. Comparison of reconstruction probabilityII of different strategies

\section{Conclusion}

In order to efficiently solve the problem of fast reconstruction of unknown sparsity of compressed signal, this paper proposes a new method of signal reconstruction, which is more adaptive and efficient. The proposed method is easy to implement, and the adopted fast estimation process can be used to efficiently deal with the unknown signal; at the same time, the performance of the proposed strategy is compared with the signal probability evaluation index for verification. The simulation results show that the performance of the proposed method will be significantly reduced if the sparsity is too large, the solution of which is the focus of future research. 


\section{References}

[1] Fira C M, Goras L, Barabasa C, et al. ECG compressed sensing based on classification in compressed space and specified dictionaries[C]// Signal Processing Conference, 2011, European. IEEE, 2011:1573-1577.

[2] Liu Z, Yang Y. Novel sparse signal reconstruction algorithm based on compressed sensing theory[J]. Journal of Optoelectronics Laser, 2011, 22(2):292-296.

[3] Zhou C, Zhao R, Hu S. An Improved Gradient Pursuit Algorithm for Signal Reconstruction Based on Compressed Sensing[C]// International Conference on Wireless Communications NETWORKING and Mobile Computing. IEEE, 2010:1-4.

[4] Bo L I, Xie J Z, Wang B L. Signal Reconstruction Based on Compressed Sensing[J]. Computer Technology \& Development, 2009.

[5] Wei X, Bao G, Ye Z, et al. Compressed sensing based underdetermined blind source separation with unsupervised sparse dictionary self-learning[C]// IEEE International Conference on Signal Processing, Communication and Computing. IEEE, 2013:1-4. 